\title{
Proton-Alpha Drift Instability of Electromagnetic Ion-Cyclotron Modes: Quasilinear Development
}

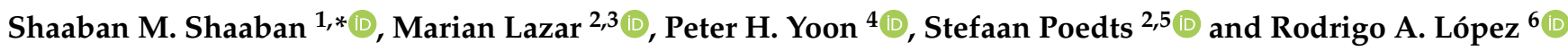 \\ 1 Theoretical Physics Research Group, Physics Department, Faculty of Science, Mansoura University, \\ Mansoura 35516, Egypt \\ 2 Centre for Mathematical Plasma Astrophysics, KU Leuven, Celestijnenlaan 200B, B-3001 Leuven, Belgium; \\ marian.lazar@kuleuven.be (M.L.); stefaan.poedts@kuleuven.be (S.P.) \\ 3 Institut für Theoretische Physik IV, Ruhr-Universität Bochum, D-44780 Bochum, Germany \\ 4 Institute for Physical Science and Technology, University of Maryland, College Park, MD 20742, USA; \\ yoonp@umd.edu \\ 5 Institute of Physics, University of Maria Curie-Skłodowska, Pl. M. Curie-Skłodowska 5, 20-031 Lublin, Poland \\ 6 Departamento de Física, Facultad de Ciencias, Universidad de Santiago de Chile, Santiago 8320000, Chile; \\ rodrigo.lopez.h@usach.cl \\ * Correspondence: s.m.shaaban88@gmail.com
}

Citation: Shaaban, S.M.; Lazar, M.; Yoon, P.H.; Poedts, S.; López, R.A. Proton-Alpha Drift Instability of Electromagnetic Ion-Cyclotron Modes: Quasilinear Development. Physics 2021, 3, 1175-1189.

https://doi.org/10.3390/ physics3040075

Received: 16 September 2021 Accepted: 23 November 2021 Published: 1 December 2021

Publisher's Note: MDPI stays neutral with regard to jurisdictional claims in published maps and institutional affiliations.

Copyright: (c) 2021 by the authors. Licensee MDPI, Basel, Switzerland. This article is an open access article distributed under the terms and conditions of the Creative Commons Attribution (CC BY) license (https:/ / creativecommons.org/licenses/by/ $4.0 /)$.

\begin{abstract}
The ability of space plasmas to self-regulate through mechanisms involving self-generated fluctuations is a topic of high interest. This paper presents the results of a new advanced quasilinear (QL) approach for the instability of electromagnetic ion-cyclotron modes driven by the relative alphaproton drift observed in solar wind. For an extended parametric analysis, the present QL approach includes also the effects of intrinsic anisotropic temperatures of these populations. The enhanced fluctuations contribute to an exchange of energy between proton and alpha particles, leading to important variations of the anisotropies, the proton-alpha drift and the temperature contrast. The results presented here can help understand the observational data, in particular, those revealing the local variations associated with the properties of protons and alpha particles as well as the spatial profiles in the expanding solar wind.
\end{abstract}

Keywords: solar wind; plasmas; instabilities; waves

\section{Introduction}

In collision-poor plasmas in space, e.g., solar wind and planetary magnetospheric environments, the dynamics of plasma particles, and implicitly their macroscopic properties, are expected to be constrained by the wave turbulence and the enhanced fluctuations, which are important components of these hot and dilute plasmas [1-3]. Very intriguing is the ability of these natural plasmas to self-regulate any deviation from kinetic isotropy, e.g., drifts, beams or anisotropic temperatures in velocity space, most likely, due to the inexorable implication of the self-generated instabilities [4-6].

In solar plasma outflows, protons (subscript $p$ ) are dominant, with a very high relative density $n_{p}>90 \%$, while alpha particles (subscript $\alpha$ ) are highly contrasting, with only $n_{\alpha}<8 \%$, and a drift relative to protons of the order of local Alfvén speed [7-9]. These relative drifts may ignite the so-called ion-beam instabilities of various electrostatic (ionacoustic) or electromagnetic (EM) modes (Alfvénic, magnetosonic). The resulting enhanced fluctuations can evolve fast enough so as to affect the regulation of ion drifts and their anisotropies [10,11], but may also contribute to the preferential heating of minor ions [5,12-14]. Their number densities, and the relative proton-alpha drift are indeed observed to decline with heliospheric distance $[15,16]$, most probably due to scattering of the beaming particles involving self-generated instabilities.

The evolution of growing fluctuations, as well as the effects of their interaction with anisotropic plasma particles, cannot be captured by a linear theory of dispersion and 
stability, but needs more elaborate modeling and investigation using quasilinear (QL) approaches or/and numerical simulations $[10,17,18]$. In this paper, an advanced quasilinear (QL) approach of the EM ion-cyclotron (EMIC) instabilities, driven by the proton-alpha relative drifts, is presented as recently predicted by linear theory [11]. Such an extensive QL analysis is apt to characterize the amplification and saturation of the growing fluctuations in time, but also the relaxation effects on macroscopic properties of ion populations, including their relative drift speed $[10,19]$. A QL approach based on velocity moments, provides a reliable and straightforward description of the EM instabilities driven by the kinetic anisotropies of plasma particles [20,21], i.e., combinations of ion beams and intrinsic temperature anisotropies, typically observed in solar wind. The evolution of the main velocity moments, such as drifting or beaming speeds, and temperature components (parallel and perpendicular to the magnetic field) is confirmed by the numerical simulations, which also show that transient deformations of the distributions fade over time, while their initial shape (e.g., bi-Maxwellian with lower drifts for drifting components) is mainly restored during the relaxation $[10,21-24]$. More elaborate QL diffusion theories attempting to reproduce transient deformations of the anisotropic distribution [25] are however complicated and restricted thus far to a limited approximation of treating the wave spectral intensity as fixed and not evolving in time, which make their implementation to fully describe the saturation of the fluctuations and relaxation of the anisotropic distribution not yet feasible.

\section{Quasilinear Kinetic Theory of Proton-Alpha Drift and Anisotropy Instabilities}

\subsection{Dispersion Relation and Wave Properties}

Below, the EMIC-like instabilities, driven either by temperature anisotropies of ion populations (Section 3.1), or an alpha-proton (small) drift (Section 3.3), or the interplay of this drift and intrinsic temperature anisotropy (Section 3.2), are examined. Let us start by overviewing the linear wave properties associated with a plasma in which the ions are made of majority protons and alpha particles as the minor species. The basic theoretical framework was already discussed in a recent paper by Rehman et al. [11], but we hereby give a brief overview for the sake of completeness. The low-frequency waves of interest satisfy the cold-plasma dispersion relation specified by

$$
\begin{aligned}
\frac{c^{2} k^{2}}{\omega_{p p}^{2}} & =\frac{\epsilon_{+} \epsilon_{-}}{\epsilon}, \\
\epsilon_{ \pm} & =\frac{\omega}{\omega \pm \Omega_{p}}+\frac{n_{\alpha}}{n_{p}} \frac{\omega \mp \Omega_{p} \pm \Omega_{\alpha}}{\omega \pm \Omega_{\alpha}}, \\
\epsilon & =\frac{\Omega_{p}^{2}}{\omega^{2}-\Omega_{p}^{2}}+\frac{n_{\alpha}}{n_{p}} \frac{\Omega_{p}^{2}}{\omega^{2}-\Omega_{\alpha}^{2}},
\end{aligned}
$$

where $\omega_{p p}=\left(4 \pi n_{p} e^{2} / m_{p}\right)^{1 / 2}, \Omega_{p}=e B_{0} / m_{p} c$, and $\Omega_{\alpha}=\Omega_{p} / 2$ represent the proton plasma frequency, proton cyclotron frequency, and the cyclotron frequency associated with the alpha-particles, respectively, $e, n_{p}, m_{p}, B_{0}$, and $c$ denoting the unit electric charge, proton number density, proton mass, ambient magnetic field intensity, and the speed of light in vacuum, respectively. In Equation (1), $\omega$ and $k$ stand for the angular frequency and the wave number, respectively. Assuming that the ambient magnetic field lies along $z$ axis, $\mathbf{B}_{0}=\hat{\mathbf{z}} B_{0}$, the wave vector may be assumed to lie in $x z$ plane without loss of generality, $\mathbf{k}=\hat{\mathbf{x}} k_{\perp}+\hat{\mathbf{z}} k_{\|}=\hat{\mathbf{x}} k \sin \theta+\hat{\mathbf{z}} k \cos \theta$, where $k_{\perp}$ and $k_{\|}$are perpendicular and parallel wave vector components with respect to the ambient magnetic field vector, $k=\left(k_{\perp}^{2}+k_{\|}^{2}\right)^{1 / 2}$ and $\theta=\cos ^{-1}\left(k_{\|} / k\right)$ being the magnitude of the wave vector and the wave propagation angle, respectively. Note that the dispersion relation (1) supports the proton-cyclotron resonance $\left(\omega \sim \Omega_{p}\right)$ and the alpha-cyclotron resonance $\left(\omega \sim \Omega_{\alpha}\right)$. Instabilities may take place in the vicinity of these cyclotron frequencies when the appropriate free energies are available.

Among the useful properties of low-frequency waves is the polarization vector. The unit electric field vector $\hat{\mathbf{e}}=\delta \mathbf{E} /|\delta \mathbf{E}|$, where $\delta \mathbf{E}$ denotes the perturbed wave electric 
field, may be defined with respect to three orthogonal vectors, $\mathbf{e}_{1}=[(\hat{\mathbf{b}} \times \hat{\mathbf{k}}) \times \hat{\mathbf{k}}] /|\hat{\mathbf{k}} \times \hat{\mathbf{b}}|$, $\hat{\mathbf{e}}_{2}=(\hat{\mathbf{b}} \times \hat{\mathbf{k}}) /|\hat{\mathbf{k}} \times \hat{\mathbf{b}}|$, and $\hat{\mathbf{e}}_{3}=\hat{\mathbf{k}}$, where $\hat{\mathbf{k}}=\mathbf{k} /|\mathbf{k}|$ and $\hat{\mathbf{b}}=\mathbf{B}_{0} /\left|\mathbf{B}_{0}\right|$. For the geometry of interest, namely, $\mathbf{B}_{0}=\hat{\mathbf{z}} B_{0}$ and $\mathbf{k}=\hat{\mathbf{x}} k \sin \theta+\hat{\mathbf{z}} k \cos \theta$, one can express $\mathbf{e}_{1}=\hat{\mathbf{t}}(\sin \theta /|\sin \theta|), \hat{\mathbf{e}}_{2}=\hat{\mathbf{a}}(\sin \theta /|\sin \theta|)$, and $\hat{\mathbf{e}}_{3}=\hat{\boldsymbol{\kappa}}$, where $\hat{\boldsymbol{\kappa}}=\hat{\mathbf{x}} \sin \theta+\hat{\mathbf{z}} \cos \theta, \hat{\mathbf{a}}=\hat{\mathbf{y}}$, and $\hat{\mathbf{t}}=\hat{\mathbf{x}} \cos \theta-\hat{\mathbf{z}} \sin \theta$. In short, the unit electric field vector can be expressed by

$$
\hat{\mathbf{e}}(\mathbf{k})=\frac{\delta \mathbf{E}_{\mathbf{k}}}{\left|\delta \mathbf{E}_{\mathbf{k}}\right|}=\frac{K \hat{\boldsymbol{\kappa}}+T \hat{\mathbf{t}}+i \hat{\mathbf{a}}}{\left(1+K^{2}+T^{2}\right)^{1 / 2}} .
$$

Thus, the polarization of the wave electric field is determined through the coefficients $K$ and $T$. If $K=\infty$, then the wave is characterized as the longitudinal mode. If either $K=0$ or $T=\infty$, then the mode is a transverse mode. Rehman et al. [11] show that these constants are determined from the dispersion relation as follows:

$$
\begin{aligned}
K & =-M \sin \theta \frac{\omega}{\Omega_{p}}, \\
T & =-M \cos \theta \frac{\omega}{\Omega_{p}}, \\
M & =\left(\frac{\omega^{2}}{\Omega_{p}^{2}} \epsilon+\frac{c^{2} k_{\|}^{2}}{\omega_{p p}^{2}}\right)^{-1}\left(\frac{\omega^{2}}{\omega^{2}-\Omega_{p}^{2}}+\frac{n_{\alpha}}{n_{p}} \frac{\omega^{2}+\Omega_{\alpha}^{2}}{\omega-\Omega_{\alpha}^{2}}\right),
\end{aligned}
$$

where $\epsilon$ is defined in Equation (1).

Another useful linear wave property is the magnitude of the group velocity,

$$
\frac{v_{g}}{c}=\frac{2 c k}{\omega_{p p}^{2}} \frac{1}{(\partial / \partial \omega)\left(c k / \omega_{p p}\right)^{2}} .
$$

Rehman et al. [11] show that the quantity $(\partial / \partial \omega)\left(c k / \omega_{p p}\right)^{2}$ is given by

$$
\begin{aligned}
R= & \omega_{p} \frac{\partial}{\partial \omega}\left(\frac{c^{2} k^{2}}{\omega_{p p}^{2}}\right)=\frac{\omega \Omega_{p}^{3}}{\left(\omega^{2}-\Omega_{p}^{2}\right)\left(\omega^{2}-\Omega_{\alpha}^{2}\right)}\left(\frac{2 c^{2} k^{2}}{\omega_{p p}^{2}} \cos ^{2} \theta+\frac{\omega^{2}}{\Omega_{p}^{2}} \epsilon\left(1+\cos ^{2} \theta\right)\right)^{-1} \\
& \times\left[\frac{2 \omega^{2}}{\omega^{2}-\Omega_{p}^{2}} \frac{\left(\omega^{2}-2 \Omega_{p}^{2}\right)\left(\omega^{2}-\Omega_{\alpha}^{2}\right)}{\Omega_{p}^{4}}\right. \\
& +\frac{2 c^{2} k^{2}\left(1+\cos ^{2} \theta\right)}{\omega_{p p}^{2}}\left(\frac{\left(\omega^{2}-\Omega_{\alpha}^{2}\right)}{\omega^{2}-\Omega_{p}^{2}}+\frac{n_{\alpha}}{4 n_{p}} \frac{\omega^{2}-\Omega_{p}^{2}}{\omega^{2}-\Omega_{\alpha}^{2}}\right) \\
& \left.+\frac{n_{\alpha}}{4 n_{p}} \frac{\omega^{2}}{\Omega_{p}^{2}} \frac{8 \omega^{4}\left(2 \omega^{2}-5 \Omega_{p}^{2}\right)+3 \Omega_{p}^{4}\left(9 \omega^{2}-2 \Omega_{p}^{2}\right)}{\Omega_{p}^{2}\left(\omega^{2}-\Omega_{p}^{2}\right)\left(\omega^{2}-\Omega_{\alpha}^{2}\right)}+\frac{2 n_{\alpha}^{2}}{n_{p}^{2}} \frac{\left(\omega^{2}-\Omega_{p}^{2}\right)\left(\omega^{2}-\Omega_{\alpha}^{2}\right)}{\Omega_{p}^{4}}\right] .
\end{aligned}
$$

\subsection{Proton-Alpha Drift and Anisotropy Instability Growth Rate}

The (quasi)-linear growth/damping rate is generally given by [26]

$$
\begin{aligned}
\gamma= & \frac{\pi}{2 \omega} \sum_{j=p, \alpha} \frac{n_{j}}{n_{0}} \frac{\omega_{p j}^{2}}{\left(1+T^{2}\right) R} \int d \mathbf{v} v_{\perp}^{2} \sum_{n=-\infty}^{\infty} \\
& \times\left\{\frac{\omega}{\Omega_{j}}\left[K \sin \theta+T\left(\cos \theta-\frac{k v_{\|}}{\omega}\right)\right] \frac{J_{n}\left(b_{j}\right)}{b_{j}}-J_{n}^{\prime}\left(b_{j}\right)\right\}^{2} \\
& \times \delta\left(\omega-n \Omega_{j}-k v_{\|} \cos \theta\right)\left(\frac{n \Omega_{j}}{v_{\perp}} \frac{\partial}{\partial v_{\perp}}+k \cos \theta \frac{\partial}{\partial v_{\|}}\right) f_{j}, \\
b_{j}= & \frac{k v_{\perp} \sin \theta}{\Omega_{j}},
\end{aligned}
$$


where $n_{0}=n_{p}+n_{\alpha}$ denotes the net ambient plasma density, $J_{n}(x)$ is the Bessel function of the first kind of order $n$, and the quantities $K, T$, and $R$ are given in Equations (3) and (5).

In ref. [11], it is assumed that both the alpha particles (" $j=\alpha$ ") and protons (" $j=p$ ") are described by drifting Maxwellian distribution functions, but in the present analysis, we extend the model to include temperature anisotropy. Hence, we keep the electrons cold, minimizing their influence, and assume that both protons and alpha particles are described by the drifting bi-Maxwellian distribution functions [27],

$$
f_{j}=\frac{1}{\pi^{3 / 2} \alpha_{\perp j}^{2} \alpha_{\| j}} \exp \left(-\frac{v_{\perp}^{2}}{\alpha_{\perp j}^{2}}-\frac{\left(v_{\|}-V_{j}\right)^{2}}{\alpha_{\| j}^{2}}\right),
$$

where $f_{j}$ is normalized to unity $\left(\int d \mathbf{v} f_{j}=1\right) ; V_{j}$ is the drifting velocity along the background magnetic field, and thermal velocities $\alpha_{\perp, \| j}$ (which may evolve in time) defined in terms of the corresponding kinetic temperatures $T_{\perp, \| j}$ are

$$
\alpha_{\perp j}=\sqrt{\frac{2 k_{B} T_{\perp j}}{m_{j}}}, \quad \alpha_{\| j}=\sqrt{\frac{2 k_{B} T_{\| j}}{m_{j}}} .
$$

The assumption of drifting bi-Maxwellian distribution functions is well supported by observations [28]. In the present discussion the temperature is defined in the unit of energy. As such, the Boltzmann constant can be set equal to unity $k_{B}=1$.

Under the assumption of drifting isotropic Maxwellian model, in ref. [11], the linear growth/damping rate (6) is derived by carrying out the velocity integration. Under the more general model (7), the same calculation as that carried out in [11], is repeated. The result is a straightforward generalization,

$$
\frac{\gamma}{\Omega_{p}}=-\sum_{j} \frac{n_{\alpha}}{n_{p}} \frac{m_{p}}{m_{\alpha}} \frac{\pi^{1 / 2}}{\left[1+(M \cos \theta)^{2}\left(\omega / \Omega_{p}\right)^{2}\right] R} \sum_{n=-\infty}^{\infty} H_{j}^{n} \eta_{j}^{n} \exp \left[-\left(\zeta_{j}^{n}\right)^{2}\right],
$$

where $m_{\alpha}=4 m_{p}$ is the alpha-particle mass; $M$ and $R$ are defined in Equations (3) and (5), respectively, and

$$
\begin{aligned}
\eta_{j}^{n} & =\frac{1}{k_{\|} \alpha_{\| j}}\left[\frac{T_{\perp j}}{T_{\| j}}\left(\omega-k_{\|} V_{j}\right)-\left(\frac{T_{\perp j}}{T_{\| j}}-1\right) n \Omega_{j}\right], \\
\zeta_{j}^{n} & =\frac{\omega-n \Omega_{j}-k_{\|} V_{j}}{k_{\|} \alpha_{\| j}}, \\
H_{j}^{n} & =\left(1+\frac{M^{2} \omega^{2}}{\Omega_{p}^{2}}\right) \frac{n^{2} \Lambda_{n}\left(\lambda_{j}\right)}{\lambda_{j}}-2\left(\lambda_{j}+M \frac{n \omega}{\Omega_{p}}\right) \Lambda_{n}^{\prime}\left(\lambda_{j}\right), \\
\Lambda_{n}(x) & =I_{n}(x) e^{-x}, \quad \lambda_{j}=\frac{k_{\perp}^{2} \alpha_{\perp j}^{2}}{2 \Omega_{j}^{2}} .
\end{aligned}
$$

Here, $I_{n}(x)$ is the modified Bessel function of the first kind of order $n$.

In the present analysis, it is assumed that the proton and alpha-particle distribution functions essentially retain their drifting bi-Maxwellian form throughout the time evolution of the instability whether it be driven by the proton-alpha relative drift or the temperature anisotropies. This is, of course, an approximation, but as already discussed in the Introduction, such an approach is well supported by validation against the numerical simulation. The time evolution of the particle distributions are controlled by the dynamical evolution of the temperature and drift velocities, $T_{\perp, \| j}$ and $V_{j}$, which is discussed below. We note that the wave energy density evolution is dictated by the QL wave kinetic equation, 


$$
\frac{\partial}{\partial t}\left(\begin{array}{c}
\left\langle\delta E^{2}\right\rangle_{\mathbf{k}} \\
\left\langle\delta B^{2}\right\rangle_{\mathbf{k}}
\end{array}\right)=2 \gamma\left(\begin{array}{c}
\left\langle\delta E^{2}\right\rangle_{\mathbf{k}} \\
\left\langle\delta B^{2}\right\rangle_{\mathbf{k}}
\end{array}\right)
$$

\subsection{Quasilinear Particle Kinetic Equation and Velocity Moment Equations}

The QL evolution of the particle velocity distribution function $f_{j}$ can be described by the general velocity space diffusion equation for the particles as given by $[19,21,26]$

$$
\frac{\partial f_{j}}{\partial t}=\frac{1}{v_{\perp}} \frac{\partial}{\partial v_{\perp}} v_{\perp}\left(D_{\perp \perp} \frac{\partial f_{j}}{\partial v_{\perp}}+D_{\perp \|} \frac{\partial f_{j}}{\partial v_{\|}}\right)+\frac{\partial}{\partial v_{\|}}\left(D_{\| \perp} \frac{\partial f_{j}}{\partial v_{\perp}}+D_{\|\|} \frac{\partial f_{j}}{\partial v_{\|}}\right),
$$

with

$$
\begin{aligned}
D_{a b}= & \left.\frac{\pi e_{j}^{2}}{m_{j}^{2}} \int d \mathbf{k} \frac{\left\langle\delta E^{2}\right\rangle_{\mathbf{k}}}{1+K^{2}+T^{2}} \sum_{n=-\infty}^{\infty}\left\{\frac{\omega}{\Omega_{j}}\left[K \sin \theta+T\left(\cos \theta-\frac{k v_{\|}}{\omega}\right)\right] \frac{J_{n}\left(b_{j}\right)}{b_{j}}-J_{(}^{\prime} b_{j}\right)\right\}^{2} \\
& \times \delta\left(\omega-k_{\|} v_{\|}-n \Omega_{j}\right) \Delta_{a} \Delta_{b}, \quad(a, b=\perp, \|), \\
\Delta_{\perp}= & \frac{n \Omega_{j}}{\omega}, \quad \Delta_{\|}=\frac{v_{\perp}}{v_{\|}} \frac{\omega-n \Omega_{j}}{\omega} .
\end{aligned}
$$

The temporal evolution of velocity moments of the distribution function, such as drift velocities of the species $j$ and their temperature components $T_{\perp j}$ and $T_{\| j}$, are given by

$$
\begin{aligned}
\frac{d V_{j}}{d t} & =\frac{\partial}{\partial t} \int d \mathbf{v} v_{\|} f_{j}, \\
\frac{d T_{\perp j}}{d t} & =\frac{m_{j}}{2} \frac{\partial}{\partial t} \int d \mathbf{v} v_{\perp}^{2} f_{j,} \\
\frac{d T_{\| j}}{d t} & =m_{j} \frac{\partial}{\partial t} \int d \mathbf{v}\left(v_{\|}-V_{j}\right)^{2} f_{j} .
\end{aligned}
$$

Upon substituting the kinetic Equation (12) to the right-hand sides of Equation (14), and taking partial derivatives, one gets:

$$
\begin{aligned}
& \frac{d V_{j}}{d t}=-\int d \mathbf{v}\left(D_{\| \perp} \frac{\partial f_{j}}{\partial v_{\perp}}+D_{\|\|} \frac{\partial f_{j}}{\partial v_{\|}}\right), \\
& \frac{d T_{\perp j}}{d t}=-m_{j} \int d \mathbf{v} v_{\perp}\left(D_{\perp \perp} \frac{\partial f_{j}}{\partial v_{\perp}}+D_{\perp \|} \frac{\partial f_{j}}{\partial v_{\|}}\right), \\
& \frac{d T_{\| j}}{d t}=-2 m_{j} \int d \mathbf{v}\left(v_{\|}-V_{j}\right)\left(D_{\| \perp} \frac{\partial f_{j}}{\partial v_{\perp}}+D_{\|\| \|} \frac{\partial f_{j}}{\partial v_{\|}}\right) .
\end{aligned}
$$

Making explicit use of the definitions for diffusion coefficients (13) as well as the drifting bi-Maxwellian distribution for $f_{j}$, defined by Equation (7), it is possible to show, after some straightforward albeit tedious mathematical manipulations, that the velocity moment kinetic equations that describe the time evolution of $V_{j}, T_{\perp j}$ and $T_{\| j}$ are given by 


$$
\begin{aligned}
\frac{d V_{j}}{d t}= & \frac{2 \pi^{3 / 2} e_{j}^{2}}{m_{j}^{2} c^{2}} \sum_{n=-\infty}^{\infty} \int_{0}^{\infty} d k k \int_{-1}^{1} d(\cos \theta) \frac{|\cos \theta|\left\langle\delta B^{2}\right\rangle_{\mathbf{k}}}{1+M^{2}\left(\omega / \Omega_{p}\right)^{2}} H_{j}^{n} \eta_{j}^{n} e^{-\left(\zeta_{j}^{n}\right)^{2}}, \\
\frac{d T_{\perp j}}{d t}= & \frac{2 \pi^{3 / 2} e_{j}^{2}}{m_{j} c^{2}} \sum_{n=-\infty}^{\infty} n \Omega_{j} \int_{0}^{\infty} d k \int_{-1}^{1} d(\cos \theta) \\
& \times \frac{|\cos \theta|}{\cos \theta} \frac{\left\langle\delta B^{2}\right\rangle_{\mathbf{k}}}{1+M^{2}\left(\omega / \Omega_{p}\right)^{2}} H_{j}^{n} \eta_{j}^{n} e^{-\left(\zeta_{j}^{n}\right)^{2}}, \\
\frac{d T_{\| j}=}{d t}= & \frac{4 \pi^{3 / 2} e_{j}^{2}}{m_{j} c^{2}} \sum_{n=-\infty}^{\infty} \int_{0}^{\infty} d k \int_{-1}^{1} d(\cos \theta) \\
& \times \frac{|\cos \theta|}{\cos \theta} \frac{\left(\omega-n \Omega_{j}-k_{\|} V_{j}\right)\left\langle\delta B^{2}\right\rangle_{\mathbf{k}}}{1+M^{2}\left(\omega / \Omega_{p}\right)^{2}} H_{j}^{n} \eta_{j}^{n} e^{-\left(\zeta_{j}^{n}\right)^{2},}
\end{aligned}
$$

where the dynamical Equation (16) are now expressed in terms of perturbed magnetic field energy density,

$$
\left\langle\delta B^{2}\right\rangle_{\mathbf{k}}=\frac{c^{2} k^{2}}{\omega^{2}}\left\langle\delta E^{2}\right\rangle_{\mathbf{k}}
$$

Equation (16) together with the wave kinetic Equation (11) describe the dynamics of the instability, whose growth rate is given by Equation (9) at each time step.

Here we should caution the readers that the assumption of drifting bi-Maxwellian velocity distribution functions for all time for both protons and alpha particles while simply calculating for the time evolution of velocity moments that define these distributions is a highly idealized approach. It is known that for an electrostatic bump-on-tail instability problem under a one-dimensional approximation, the quasilinear theory predicts a local deformation of the resonant range of velocity space that leads to the velocity space plateau formation. For an electromagnetic instability driven by the temperature anisotropy, on the other hand, it is also known that the quasilinear diffusion takes place, not along the parallel velocity space, but rather along a circularly path centered around the wave phase speed, which leads to the pitch-angle space diffusion and the resultant isotropization of the initial anisotropic temperatures [29,30]. For the present problem of EMIC instability driven by either the proton-alpha relative drift or the temperature anisotropies, the situation is more akin to the pitch-angle diffusion saturation picture as discussed by the abovereferenced early literature rather than the velocity space plateau formation. Besides, recent papers by Harding et al. [31], Melrose et al. [32] discuss that the velocity plateau formation is relevant only for strictly one-dimensional problems and that for three-dimensional situations, the quasilinear process involved in the bump-on-tail instability also leads to the pitch-angle diffusion, which leads to the isotropic velocity distribution function. We thus believe that our assumption of the drifting bi-Maxwellian velocity distribution functions with time-varying velocity moments are justified.

\section{Numerical Results}

In order to aid the numerical analysis Equations (11) and (16) are considered in dimensionless form. Thus, the normalized quantities equations of relevance are:

$$
\begin{aligned}
\tau & =\Omega_{p} t, \quad x=\frac{\omega}{\Omega_{p}}, \quad q=\frac{c k}{\omega_{p p}}, \\
\beta_{\perp, \| j} & =\frac{8 \pi n_{j} T_{\perp, \| j}}{B_{0}^{2}}, \quad \beta_{0 j}=\frac{4 \pi n_{p} m_{p} V_{j}^{2}}{B_{0}^{2}}, \quad W(x, \theta) d q=\frac{4 \pi n_{p} e^{2}}{m_{p} c^{2}} \frac{\left\langle\delta B^{2}\right\rangle_{\mathbf{k}}}{B_{0}^{2}} d k,
\end{aligned}
$$

which stand for non-dimensional time, normalized wave frequency, normalized wave number, perpendicular and parallel beta's as well as the squared dimensionless drift speed for each species, and normalized wave magnetic field energy density. We solved the set of equations by numerical means. The basic time stepping method is the standard leap frog scheme. In the numerical analysis, the wave spectrum was separated into the forward 
$\left(\theta>90^{\circ}\right)$ and backward $\left(90^{\circ}<\theta<180^{\circ}\right)$ components since the finite drift velocity breaks the symmetry associated with the forward versus backward wave propagation directions.

Below in this Section, a parametric analysis of the unstable modes, obtained as numerical exact solutions of the system of QL equations, are given. First, simple cases of instabilities, driven by temperature anisotropies of protons and alpha particles, are considered, and, then, the complexity of the study is gradually increased by introducing the cumulative effect of the alpha drift velocity.

\subsection{Proton and Alpha Temperature Anisotropy-Driven Cyclotron Instabilities}

In order to test the QL derivations obtained here, let us start with three cases describing cyclotron instabilities, driven either by the anisotropic protons with $A_{p} \equiv T_{\perp p} / T_{\| p}=2$ (case 1). or by the anisotropic alpha particles $A_{\alpha}=4$ (case 2), or, cumulatively, by the protons with $A_{p}=2$ and alpha particles with $A_{\alpha}=4$ (case 3). The input parameters are as follows:

- $\quad$ case 1: $A_{p}=2, \beta_{\| p}=2, A_{\alpha}=1, \beta_{\| \alpha}=0.2$;

- $\quad$ case 2: $A_{p}=1, \beta_{\| p}=2, A_{\alpha}=4, \beta_{\| \alpha}=0.2$;

- case 3: $A_{p}=2, \beta_{\| p}=2, A_{\alpha}=4, \beta_{\| \alpha}=0.2$.

Common plasma parameters in these cases are $n_{\alpha} / n_{p}=0.05, T_{\| \alpha} / T_{\| p}=2$, and $\beta_{0 p, \alpha}=V_{p, \alpha}^{2} / v_{A}^{2}=0$.

Figure 1 presents the results of the QL temporal evolution for the enhanced magnetic fluctuations $\delta B^{2} / B_{0}^{2}=\int d \mathbf{k}\left(\delta B_{\mathbf{k}} / B_{0}\right)^{2}$ (top row), and their effects on the macroscopic plasma parameters, i.e., proton plasma betas $\beta_{\perp, \| p}$ (middle), alpha plasma betas $\beta_{\perp, \| \alpha}$ (bottom), for the initial plasma parameters corresponding to case 1 (left), 2 (middle), and 3 (right).
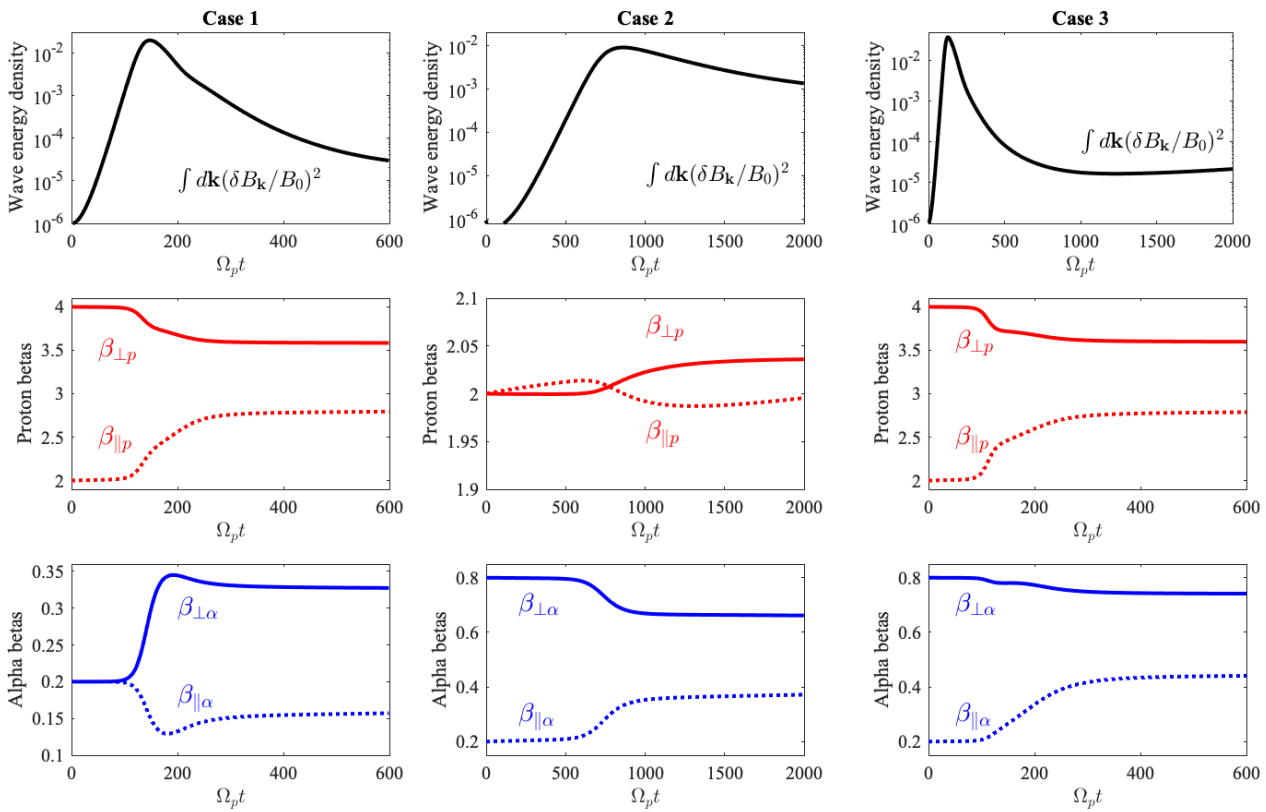

Figure 1. Quasilinear (QL) temporal variations for cases 1-3: the magnetic field energy density (top row), plasma beta parameters for protons (middle row), and alpha particles (bottom row). See text for details.

For case 1 (left column), the proton cyclotron instability is driven by the initially anisotropic protons $A_{p}(0)=2$, and enhances the magnetic wave fluctuations $\delta B^{2} / B_{0}^{2}$. As a direct consequence, protons are subjected to perpendicular cooling and parallel heating, as indicated by, respectively, the perpendicular (red solid line) and parallel (red dashed line) components of the proton beta parameter $\beta_{\perp, \| p}$. On the other hand, initially isotropic $\left[A_{\alpha}(0)=1\right]$ alpha particles are subjected to perpendicular heating and parallel cooling as 
shown by, respectively, the perpendicular (blue solid line) and parallel (blue dashed line) alpha beta parameters.

For case 2 , the initially anisotropic alpha particles $\left[A_{\alpha}(0)=4\right]$ excite the alpha cyclotron instability. The enhanced wave fluctuations $\left(\delta B^{2} / B_{0}^{2}\right)$ determine a perpendicular cooling and parallel heating of alpha particles, as reflected by the perpendicular (blue solid line) and parallel (blue dashed line) alpha beta parameters $\beta_{\perp, \| \alpha}$, respectively. In this case plasma beta parameters of the initially isotropic protons experience perpendicular heating (red solid line) and parallel cooling (red dashed line).

In case 3, plasma beta parameters for protons and alphas are subjected to perpendicular cooling and parallel heating by the enhanced wave fluctuations. The interplay of protons and alpha particles temperature anisotropies inhibits the perpendicular cooling of alpha particles-see the bottom right panel. It is worth noting that all these results are in agreement with those reported in [10] for different plasma conditions.

Figure 2 summarizes the discussion on the cooling and heating of plasma ions by the means of temperature anisotropy for protons (red) and alpha particles (blue) $A_{p, \alpha} \equiv \beta_{\perp} / \beta_{\|}$ in the left panels, and alpha-proton parallel temperature ratio $T_{\| \alpha} / T_{\| p}$ in the right panels. For case 1, the temperature anisotropy of protons is relaxed as a function of time $\left(\tau=\Omega_{p} t\right)$, while the initially isotropic alpha particles ended up with large temperature anisotropy in the perpendicular direction $A_{\alpha}\left(\tau_{\max }\right)>1$. An opposite situation is observed in case 2, the temperature anisotropy of alpha particles is relaxed in time, while the initially isotropic protons gain small anisotropy in perpendicular direction $A_{p}\left(\tau_{m}\right) \gtrsim 1$. For case 3 , both proton and alphas anisotropies are relaxed, under action of the enhanced magnetic wave fluctuations of the accumulated proton and alpha cyclotron instabilities. Moreover, a relaxation for the alpha-proton temperature ratio $T_{\| \alpha} / T_{\| p}$ is observed associated only with the excitation of the proton cyclotron instability (case 1), while in the other two cases (cases 2 and 3) one finds an enhancement of this temperature contrast mainly determined by the anisotropic alpha particles.
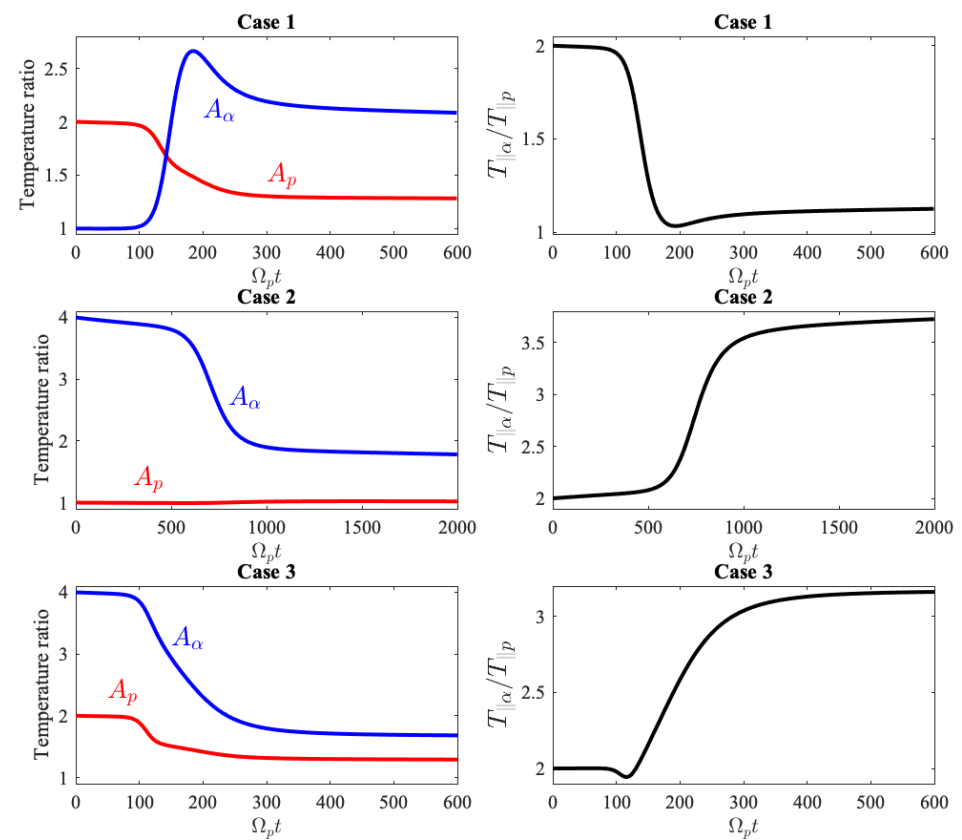

Figure 2. Temporal evolution for cases 1-3: temperature anisotropies (left column) and alpha-proton temperature ratio (right column). See text for details.

\subsection{Interplay of Temperature Anisotropies and Alpha-Proton Drifts}

Here, the complexity of the analysis is increased by considering a finite drift velocity of alpha particles (parallel to the background magnetic field) as an additional source of free energy. In order to show the effects induced by the drift, the same initial plasma parameters 
as in cases 1, 2 and 3 are considered, but with finite alpha drifts, which here are named cases 4,5 , and 6 , respectively:

- $\quad$ case 4: $A_{p}=2, A_{\alpha}=1, \beta_{0 \alpha}=\frac{V_{\alpha}^{2}}{v_{A}^{2}}=1$;

- $\quad$ case 5: $A_{p}=1, A_{\alpha}=4, \beta_{0 \alpha}=\frac{V_{\alpha}^{2}}{v_{A}^{2}}=0.25$;

- $\quad$ case 6: $A_{p}=2, A_{\alpha}=4, \beta_{0 \alpha}=\frac{V_{\alpha}^{2}}{v_{A}^{2}}=1$.

Figure 3 shows the effects of the drift velocity of alpha particles on the temporal profiles of the macroscopic plasma parameters associated with the excitation of the proton cyclotron instability (case 4, left column), alpha cyclotron instability (case 5, middle column), and proton and alpha cyclotron cumulative instabilities (case 6, right column). Similar to case 1 , the temporal evolution of the proton plasma beta parameters in case 4 are not affected by the drift velocity of alpha particles. Both alpha plasma beta parameters are increased in time, but the alphas are heated more in perpendicular direction, and become anisotropic at the final stage, that is, $A_{\alpha}\left(\tau_{m}\right)>1$. For case 5, only parallel plasma beta parameter for protons is subjected to heating for a finite alpha drift. The interplay of the drift velocity and anisotropy of alpha particles enhance the cooling and heating mechanisms for the perpendicular and parallel alpha plasma betas, respectively; and alpha particles become less anisotropic, compared to case 2 . For case 6 , the interplay of three sources of free energies is considered, i.e., temperature anisotropies of protons and alphas, and the drift velocity of alpha particles. In this case, there are two opposite effects on the temporal profiles of the alpha plasma parameters (the first effect is already shown in Figure 1), such that temperature anisotropy inhibits the relaxation of the temperature anisotropy of alpha particles in the perpendicular direction, while the second effect is induced by the alpha drift velocity, markedly stimulating the cooling and heating mechanisms on alpha particles, which become isotropic at later time, i.e., $A_{\alpha}(\tau) \sim 1$. By comparing cases 5 and 6 , one can state that the isotropization of the alpha particles is markedly enhanced with increasing the drift velocity, i.e., for $V_{\alpha} / v_{A}=0.5$ in case 5 , and $V_{\alpha} / v_{A}=1$ in case 6 .

Figure 4 summarizes the relaxation of the relative drift and the induction of temperature anisotropies of proton and alpha particles (left), and the alpha-proton temperature ratio (right). By contrasting with results in Figure 2, one can extract some effects induced by a finite alpha drift velocity. In case 4 , the relaxation of proton anisotropy is not affected by the drift velocity of alpha particles, but the induced temperature anisotropy of alpha particles is much lower in case 4 than that in case 1. Furthermore, the relaxation of the alpha-proton temperature ratio is lower than that in case 1. For case 5, the alpha drift velocity slightly enhances the relaxation of alpha particles compared to case 2 (with $V_{\alpha}=0$ ). In case 5 , the initially isotropic protons remain nearly isotropic over time $A_{p}\left(\tau_{m}\right) \lesssim 1$. In case 6 , one can see the relaxation of the proton anisotropy is comparable to that in case 3 . However, the presence of alpha drift velocity markedly enhances the relaxation of the anisotropy of alpha particles, which become isotropic at final stage $A_{\alpha}\left(\tau_{m}\right) \sim 1$. In cases 5 and 6 , the induced alpha-proton temperature ratios are enhanced by the initial drift of alpha particles. In all cases drift velocities are relaxed in time. It is worth to note that relaxation of the proton anisotropy and alpha drift velocity, and the induction of the alpha temperature anisotropy in case 4 (top left panel) are in good agreement with those obtained from the two-dimensional hybrid simulation reported by [33] for different plasma conditions, see Figure 1 therein. 

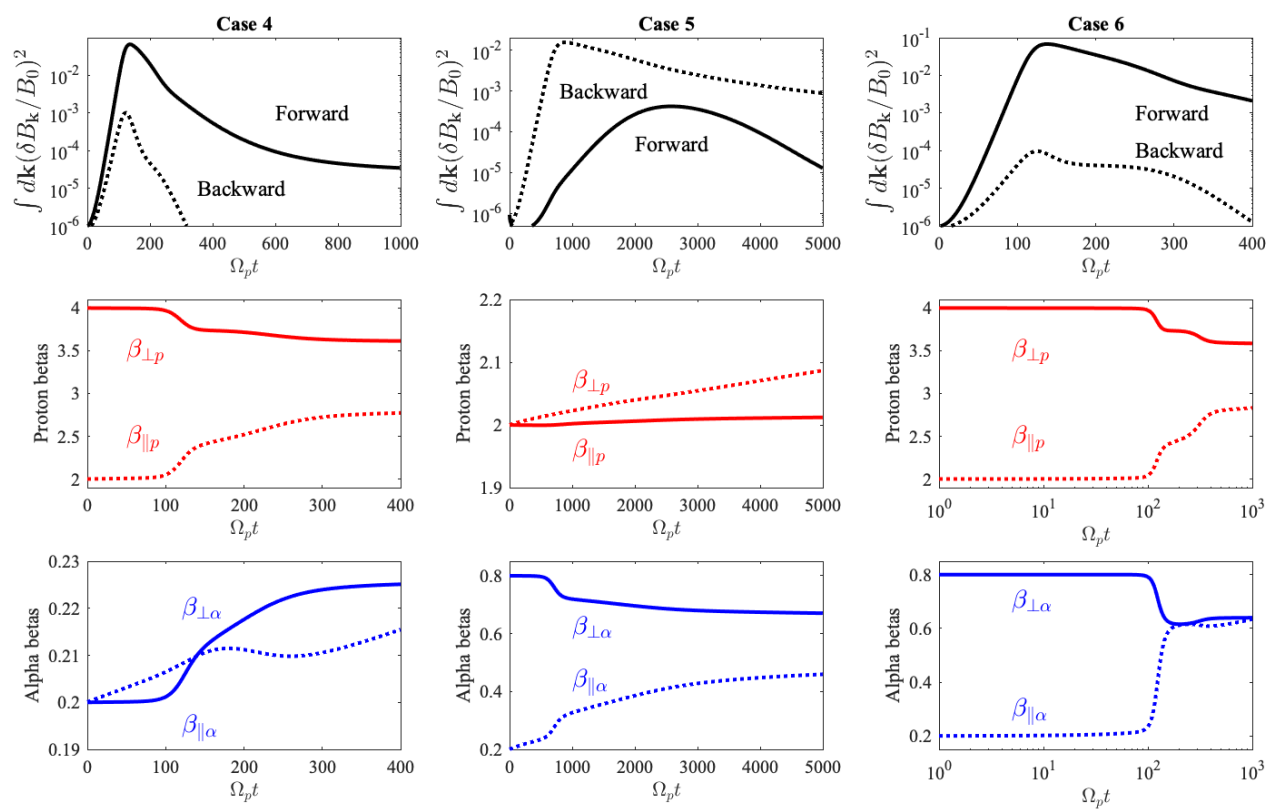

Figure 3. QL dynamical evolution for cases 4-6: the magnetic wave energy density (top row), plasma beta parameters for protons (middle row), and alpha particles (bottom row). See text for details.
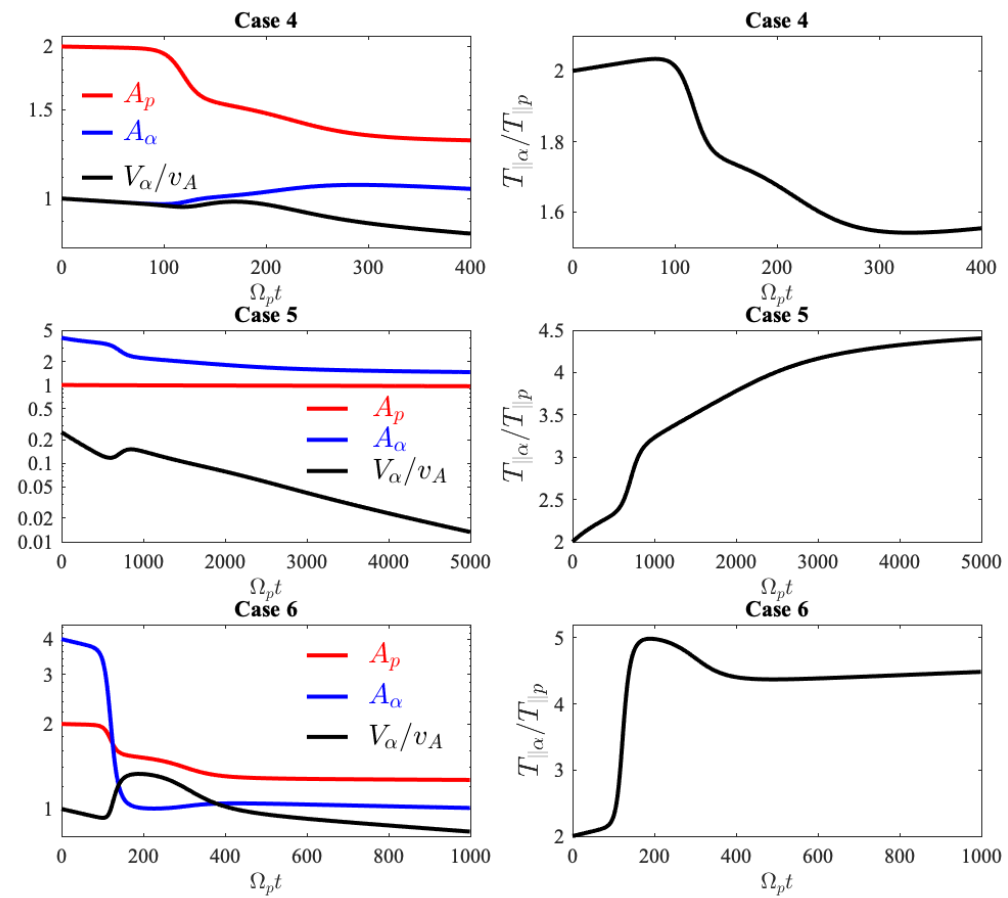

Figure 4. The relaxation of the drift velocity and the induced temperature anisotropies (left column), and alpha-proton temperature ratio (right column) for cases 5 and 6.

\subsection{Instability Driven by the Alpha-Proton Drift (Isotropic Temperatures)}

In this Section, it is assumed that the protons and alpha particles are initially isotropic, i.e., $A_{\alpha, p}(0)=1$, and study the alpha cyclotron instability driven by the alpha drift velocity, and its consequences on the plasma particles. This case is summarized by the following initial parameters:

- $\quad$ case 7: $A_{p}=1, \beta_{\| p}=0.05, A_{\alpha}=1, \beta_{\| \alpha}=0.005, \beta_{0 p}=\frac{V_{p}^{2}}{v_{A}^{2}}=0, \beta_{0 \alpha}=\frac{V_{\alpha}^{2}}{v_{A}^{2}}=4$.

Figure 5 displays the temporal profiles for wave energy associated with the enhanced fluctuations (top-left). In this case the beaming cyclotron instability is driven by finite 
alpha-proton drift. Figure 5 also plots the plasma beta parameters of protons (bottom-left) and alpha particles (top-right), as well as their temperature anisotropies and drift velocity (bottom-right). The enhanced fluctuations are evident only for the forward propagating modes, meaning that the beam alpha cyclotron instability is excited in the direction parallel to the background magnetic field vector. Both components of plasma betas for proton and alpha particles are increased in time, i.e., as $\tau=\Omega_{p} t$ increases, but both species are heated more along the perpendicular direction. Bottom-right panel of Figure 5 summarizes the consequences of the enhanced fluctuations, presenting the temperature anisotropies of protons (red) and alpha particles (blue), and alpha drift velocity (black). Both species gain energy in perpendicular direction and become anisotropic at later stages with $A_{\alpha, p}\left(t_{m}\right)>1$. However, the induced temperature anisotropy of alpha particles is much larger than that gained by the protons. The drift velocity is relaxed as $\tau$ increases. These results are in good agreement with those obtained recently from 2.5D and 3D hybrid simulations for different plasma conditions [22]; see Figure 1 in [22].
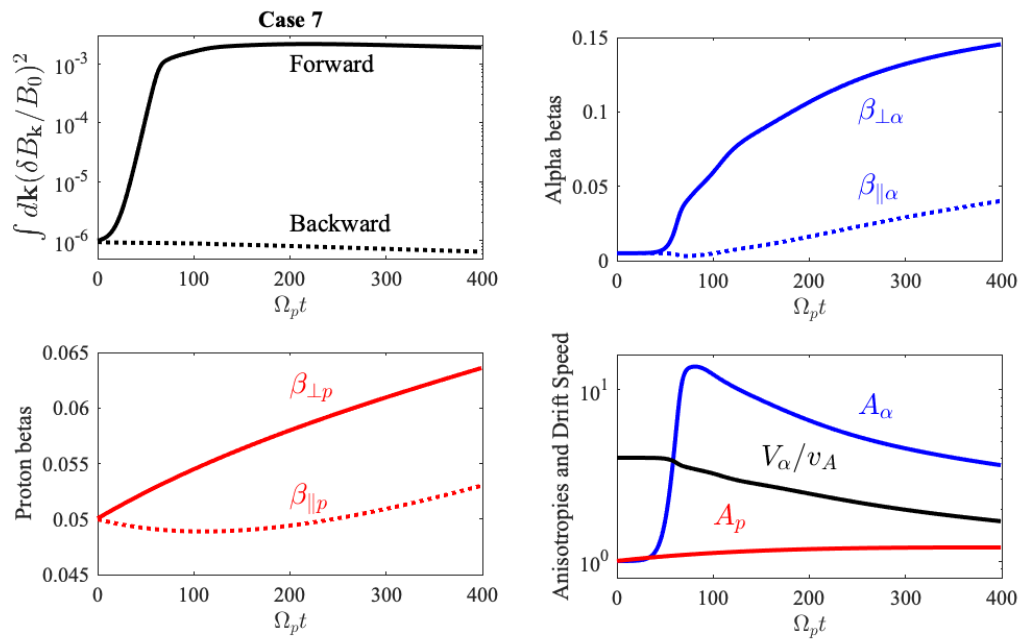

Figure 5. Case 7: QL dynamical evolution of the magnetic wave energy density (top left panel), plasma beta parameters for protons (bottom left panel), and alpha particles (top right panel), and for relaxing the drift velocity and inducing temperature anisotropies (bottom right panel). See text for details.

For the same plasma parameters as in case 7 , but for different initial plasma beta parameters, the QL analysis is carried out further on:

- $\quad$ case 8: $\beta_{p}=0.1, \beta_{\alpha}=0.01, \beta_{0 \alpha} \in\left[1,2, d \beta_{0 \alpha}=0.25\right]$;

- $\quad$ case 9: $\beta_{p}=0.5, \beta_{\alpha}=0.05, \beta_{0 \alpha} \in\left[1,2, d \beta_{0 \alpha}=0.25\right]$;

- case 10: $\beta_{p}=1.5, \beta_{\alpha}=0.15, \beta_{0 \alpha} \in\left[1,2, d \beta_{0 \alpha}=0.25\right]$.

The result is given Figure 6, where the QL dynamical evolution of the alpha drift velocity (top), temperature anisotropies of protons (middle row) and alpha particles (bottom row) as functions of $\beta_{\| p}(\tau)$ (or $\beta_{\| \alpha}(\tau)$ ) for different initial plasma betas $\beta_{\| p}(0)=0.1$ (case 8 , left column), $\beta_{\| p}(0)=0.5$ (case 9, middle column), and $\beta_{\| p}(0)=1.5$ (case 10, right column) are displayed. For each case the evolutions for five different initial values of the alpha drift velocity $\beta_{0 \alpha} \in\left[1,2, d \beta_{0 \alpha}=0.25\right]$ are computed.

Top panels of Figure 6 show the relaxation of alpha drift velocity as a function of $\beta_{\| p}(\tau)$. The alpha-proton relative drift speed decreases to a very low level, i.e., $V_{\alpha} / V_{A} \rightarrow 0$, suggesting a complete relaxation of ion beams. Physically, ions can indeed be scattered by the resulting fluctuations, contributing to their redistribution in velocity space. In all these cases, the relaxation of the drift velocity is associated with a heating of protons in parallel direction. Middle panels show the dynamical evolution of the proton temperature anisotropy as a function of $\beta_{\| p}(\tau)$. 
These dynamical evolution can be divided into two regimes, the first regime corresponds to low plasma betas, $\beta_{\| p} \sim 0.1$, when protons are slightly heated in the perpendicular direction and become anisotropic with $A_{p}>1$, confirming the results in Figure 5. The second regime starts by increasing $\beta_{\| p}>0.1$ (cases 9 and 10), or when the dynamical evolution of beta exceeds $\beta_{\| p}(\tau)>0.1$. Increasing $\beta_{\| p}$ enhances the proton heating in parallel direction, and protons become (parallel) anisotropic at later stages with $A_{p}\left(\tau_{m}\right)<1$. The same effects are observed for alpha particles in the bottom panels.

The dynamical evolution of the temperature anisotropy of alpha particles as a function of $\beta_{\| \alpha}(\tau)$ can also be divided into two regimes. For low betas $\beta_{\| \alpha}<0.1$, the alpha particles are subjected to heating and cooling in the perpendicular and parallel directions, respectively, and alphas become anisotropic at later stages with large anisotropy in perpendicular direction $A_{\alpha}>1$, confirming the results in Figure 5. The induced temperature anisotropies of alpha particles $A_{\alpha}>1$ are in general increasing as the drift velocity increases. The second regime starts beyond $\beta_{\alpha}=0.1$, when alpha particles gain (induced) temperature anisotropies only in parallel direction, i.e., $A_{\alpha}<1$.
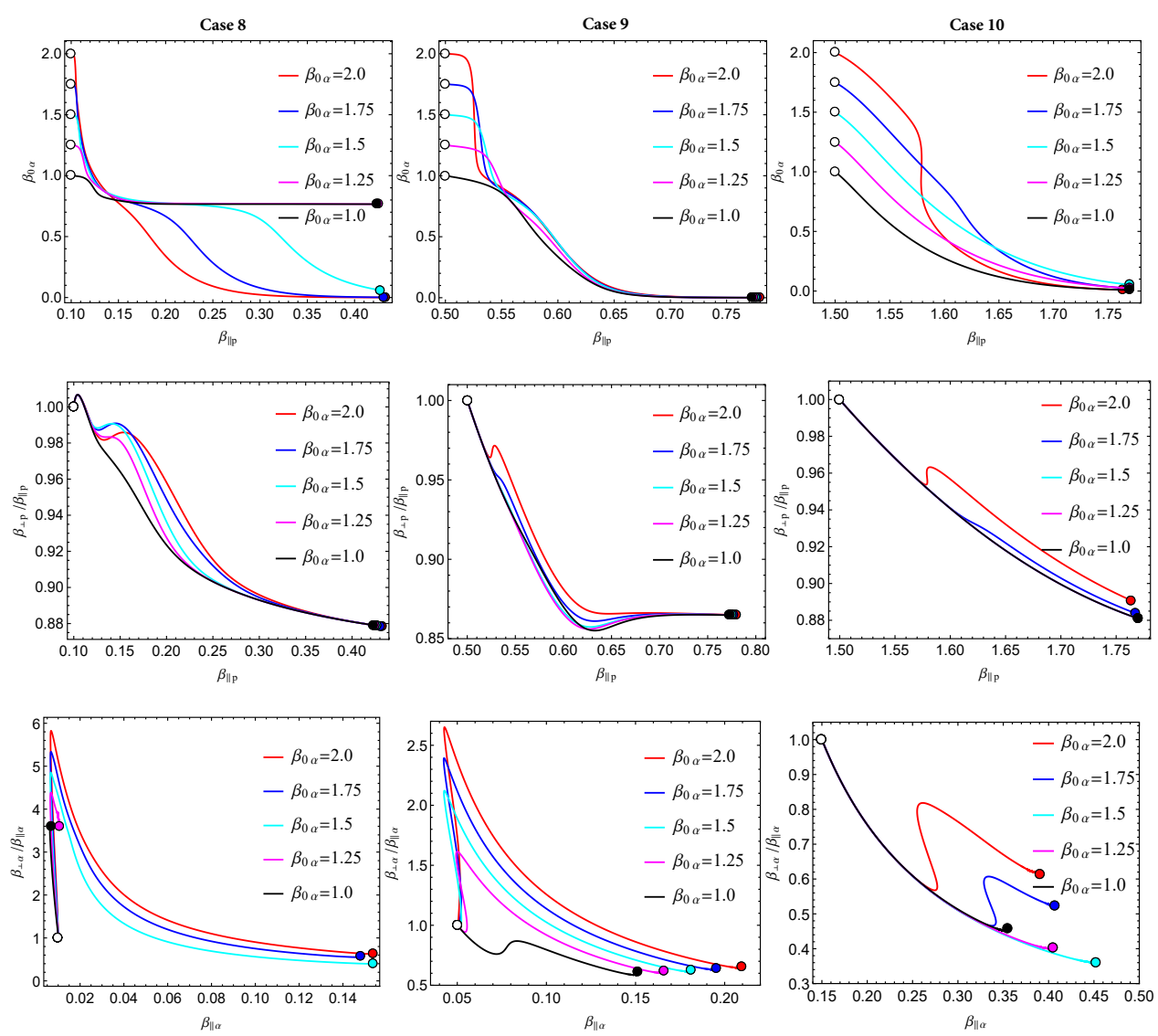

Figure 6. Dynamical evolution of the alpha drift velocity (top row), temperature anisotropies of protons (middle row) and alpha particles (bottom row) as functions of $\beta_{\| p}(\tau)$ (or $\beta_{\| \alpha}(\tau)$ ) for cases 8 (left column), 9 (middle column), and 10 (right column). See text for details.

\section{Conclusions}

In this paper, an advanced quasilinear (QL) analysis of the electromagnetic ioncyclotron (EMIC) instabilities, driven by the kinetic anisotropies of protons and alpha particles, i.e., their relative drift, combined with or without the intrinsic temperature anisotropies, is presented. Such plasma conditions are specific to the solar atmosphere at short heliosphere distances, in the outer corona and solar wind. The long term evolution of the growing fluctuations, triggered by cyclotron instabilities, and also the consequences of their interaction were characterized with plasma particles, i.e., protons and alpha particles. 
The paper presents for the first time the consequences of both the forward and backward fluctuations on the relaxation of the drift and temperature anisotropy of alpha and protons. Comparing the results obtained here with those of [33], where the analysis refers to total fluctuations, we were able to study the consequences of the backward fluctuations. The nonuniform relaxation of the macroscopic quantities in Figures 4 and 6 can be explained as a result of the competition between the backward and forward propagating modes and their enhanced fluctuations, as explained in [33] based on the hybrid simulations, e.g., in [22].

The results of the parametric study in Section 3 describe the effects from the interplay of the alpha-proton relative drift velocity and their temperature anisotropies on the saturation of the self-generated cyclotron instabilities and the relaxation of the non-thermal distributions. For a non-drifting scenario (Figures 1 and 2), the enhanced EMIC fluctuations are triggered by temperature anisotropy of protons or alphas, and on long term, show multiple effects on the particles. In addition to a relaxation of the anisotropy to quasi-stable states (below the thresholds of instability), the induction of a temperature anisotropy (in a perpendicular direction) to the other initially isotropic species are also observed. The induced temperature anisotropy of alpha particles is in general much larger than that of the protons. It is also found that the interplay of temperature anisotropies of protons and alpha particles has an inhibiting effect on the perpendicular cooling of alpha particles during the relaxation. Moreover, the alpha-proton temperature contrast $\left(T_{\| \alpha} / T_{\| p}\right)$ is reduced only during the excitation of the proton cyclotron instability, but it is enhanced in the presence of the alpha instability fluctuations.

Comparing to the non-drifting scenario, an initial, relatively small alpha drift velocity stimulates the enhanced fluctuations and implicitly the relaxation of the alpha temperature anisotropy (Figures 3 and 4). The relaxation of temperature anisotropy is markedly enhanced with the increasing drift velocity of alpha particles, whose temperature becomes isotropic at final stage $A_{\alpha} \sim 1$. One the other hand, in the generation of the proton cyclotron fluctuations the relaxation of the proton anisotropy is not affected by the alpha drift velocity, but the induced temperature anisotropy of alpha particles is much lower than that in the non-drifting scenario. In all the cases drift velocities are relaxed as time evolves, and temporal profiles of the alpha-proton temperature contrast $\left(T_{\| \alpha} / T_{\| \alpha}\right)$ are in general similar to those obtained for the non-drifting populations. However, a finite alpha drift may lead to an increase of this contrast in the excitation of the alpha cyclotron instability.

In Section 3.3, the QL temporal evolution of the beam alpha cyclotron instability, driven by the alpha-proton relative drift, is described with both protons and alpha particles considered initially isotropic (Figure 5). The enhanced fluctuations are associated only with the forward propagating modes, parallel to the background magnetic field. These fluctuations act back on the particles, reducing their drift velocity as time evolves. Concomitantly, the instability effectively leads to the perpendicular heating of the protons and alphas, so that at later stages, both species exhibit the perpendicular temperature anisotropies, $A_{p, \alpha}>1$. The induced temperature anisotropy of alpha particles is in general much larger than that gained by protons. Figure 6 displays the QL dynamical evolution of the macroscopic plasma parameters, i.e., alpha drift velocity, temperature anisotropies of alphas and protons, and their temperature contrast, as a function of the parallel plasma betas $\beta_{\| \alpha, p}$. The dynamical evolution shows the relaxation of the drift velocity being associated with a heating of protons in parallel direction. One can distinguish two regimes conditioned by the parallel plasma betas $\beta_{\| \alpha, p}$ in this dynamical evolution of the temperature anisotropies of protons and alpha particles. First, for $\beta_{\| \alpha, p}<0.1$, protons and alpha particles are heated in the perpendicular direction and become anisotropic with $A_{p, \alpha}>1$. The second regime starts beyond $\beta_{\| \alpha, p}=0.1$, when both protons and alpha particles gain induced anisotropy in parallel direction, i.e., $A_{p, \alpha}<1$. These results are in good agreement with those obtained from hybrid and PIC simulations reported in the literature, see for instance $[10,22,33]$.

To conclude, the study shows that the interplay of different sources of free energy present in solar wind has an important impact on the enhanced fluctuations, in particular those triggered by the EMIC instabilities, which in turn contribute to an exchange of 
energy between proton and alpha particles, leading to important variations of the temperature anisotropies, the proton-alpha drift and the temperature contrast. Future studies should also consider complementary conditions of more energetic beams (with higher drifts), which may excite different (e.g., firehose-like, or electrostatic) instabilities, possibly, with different consequences on the relaxation of the populations involved, especially, on their temperatures [34]. The results obtained here clearly show that self-generated EMIC instabilities can contribute to the regulation of drifts and anisotropies of ions present in the solar wind. Note that the QL theory, considered here, contains both parallel and perpendicular unstable solutions, although we do not give the detailed two-dimensional spectral characteristics associated with the unstable mode. Recently, Liu et al. [35] investigated the conditions when oblique instabilities are more competitive. In the future, it will be interesting to compare the QL theory here presented with the findings in [35] by analyzing the detailed 2D wave spectrum, but this is beyond the scope of the present study.

Author Contributions: The authors contributions are equal. All authors have read and agreed to the published version of the manuscript.

Funding: The authors acknowledge support from the Katholieke Universiteit Leuven, Ruhr-University Bochum, and Christian-Albrechts University Kiel. S. M. Shaaban acknowledges the Alexander-vonHumboldt Research Fellowship. R.A. López acknowledges the support of ANID Chile through FONDECyT grant No. 11201048. These results were also obtained in the framework of the projects C14/19/089 (C1 project Internal Funds KU Leuven), G.0D07.19N (FWO-Vlaanderen), SIDC Data Exploitation (ESA Prodex-12), and Belspo projects BR/165/A2/CCSOM and B2/191/P1/SWiM. P.H. Yoon acknowledges NASA Grant NNH18ZDA001N-HSR and NSF Grant 1842643 to the University of Maryland.

Data Availability Statement: The data that support the findings of this study are available from the corresponding author upon request.

Conflicts of Interest: The authors declare no conflict of interest.

\section{References}

1. Alexandrova, O.; Chen, C.H.K.; Sorriso-Valvo, L.; Horbury, T.S.; Bale, S.D. Solar wind turbulence and the role of ion instabilities. Space Sci. Rev. 2013, 178, 101-139. [CrossRef]

2. Woodham, L.D.; Wicks, R.T.; Verscharen, D.; Owen, C.J.; Maruca, B.A.; Alterman, B.L. Parallel-propagating fluctuations at proton-kinetic scales in the solar wind are dominated by kinetic instabilities. Astrophys. J. 2019, 884, L53. [CrossRef]

3. Bowen, T.A.; Mallet, A.; Huang, J.; Klein, K.G.; Malaspina, D.M.; Stevens, M.; Bale, S.D.; Bonnell, J.W.; Case, A.W.; Chandran, B.D.G.; et al. Ion-scale electromagnetic waves in the inner heliosphere. Astrophys. J. Suppl. Ser. 2020, 246, 66. [CrossRef]

4. Bale, S.D.; Kasper, J.C.; Howes, G.G.; Quataert, E.; Salem, C.; Sundkvist, D. Magnetic fluctuation power near proton temperature anisotropy instability thresholds in the solar wind. Phys. Rev. Lett. 2009, 103, 211101. [CrossRef] [PubMed]

5. Matteini, L.; Hellinger, P.; Goldstein, B.E.; Landi, S.; Velli, M.; Neugebauer, M. Signatures of kinetic instabilities in the solar wind. J. Geophys. Res. Space Phys. 2013, 118, 2771-2782. [CrossRef]

6. Sun, H.; Zhao, J.; Xie, H.; Wu, D. On kinetic instabilities driven by ion temperature anisotropy and differential flow in the solar wind. Astrophys. J. 2019, 884, 44. [CrossRef]

7. Asbridge, J.R.; Bame, S.J.; Feldman, W.C.; Montgomery, M.D. Helium and hydrogen velocity differences in the solar wind. J. Geophys. Res. 1976, 81, 2719-2727. [CrossRef]

8. Neugebauer, M. Observations of solar-wind helium. Fundam. Cosm. Phys. 1981, 7, 131-199. [CrossRef]

9. Marsch, E. Kinetic physics of the solar corona and solar wind. Living Rev. Sol. Phys. 2006, 3, 1. [CrossRef]

10. Yoon, P.H.; Seough, J.; Hwang, J.; Nariyuki, Y. Macroscopic quasi-linear theory and particle-in-cell simulation of helium ion anisotropy instabilities. J. Geophys. Res. Space Phys. 2015, 120, 6071-6084. [CrossRef]

11. Rehman, M.A.; Shaaban, S.M.; Yoon, P.H.; Lazar, M.; Poedts, S. Electromagnetic instabilities of low-beta alpha/proton beams in space plasmas. Astrophys. Space Sci. 2020, 365, 107. [CrossRef]

12. Maneva, Y.G.; Araneda, J.A.; Marsch, E. Regulation of ion drifts and anisotropies by parametrically unstable finite-amplitude Alfvén-cyclotron waves in the fast solar wind. Astrophys. J. 2014, 783, 139. [CrossRef]

13. Verscharen, D.; Chandran, B.D.G.; Bourouaine, S.; Hollweg, J.V. Deceleration of alpha particles in the solar wind by instabilities and the rotational force: Implications for heating, azimuthal flow, and the Parker spiral magnetic field. Astrophys. J. 2015, 806, 157. [CrossRef]

14. Markovskii, S.A.; Chandran, B.D.G.; Vasquez, B.J. Ion heating resulting from the deceleration of alpha particles by a proton-alpha drift instability in a nonuniform solar-wind plasma. Astrophys. J. 2019, 870, 121. [CrossRef] 
15. Marsch, E.; Mühlhäuser, K.H.; Rosenbauer, H.; Schwenn, R.; Neubauer, F.M. Solar wind helium ions: Observations of the Helios solar probes between 0.3 and 1 AU. J. Geophys. Res. Space Phys. 1982, 87, 35-51. [CrossRef]

16. Neugebauer, M.; Goldstein, B.E.; Smith, E.J.; Feldman, W.C. Ulysses observations of differential alpha-proton streaming in the solar wind. J. Geophys. Res. Space Phys. 1996, 101, 17047-17055. [CrossRef]

17. Yoon, P.H.; Schlickeiser, R. On the equilibrium between proton distribution and compressible kinetic Alfvénic fluctuations. Mon. Not. R. Astron. Soc. 2018, 482, 4279-4289. [CrossRef]

18. Shaaban, S.M.; Lazar, M.; Schlickeiser, R. Electromagnetic ion cyclotron instability stimulated by the suprathermal ions in space plasmas: A quasi-linear approach. Phys. Plasmas 2021, 28, 022103. [CrossRef]

19. Yoon, P.H.; Seough, J. Quasilinear theory of anisotropy-beta relation for combined mirror and proton cyclotron instabilities J. Geophys. Res. Space Phys. 2012, 117, A08102. [CrossRef]

20. Davidson, R.C.; Völk, H.J. Macroscopic quasilinear theory of the Garden-Hose instability. Phys. Fluids 1968, 11, 2259-2264. [CrossRef]

21. Yoon, P.H. Kinetic instabilities in the solar wind driven by temperature anisotropies. Rev. Mod. Plasma Phys. 2017, 1, 4. [CrossRef]

22. Ofman, L. Nonlinear Evolution of ion kinetic instabilities in the solar wind. Sol. Phys. 2019, 294, 51. [CrossRef]

23. López, R.A.; Shaaban, S.M.; Lazar, M.; Poedts, S.; Yoon, P.H.; Micera, A.; Lapenta, G. Particle-in-cell simulations of the whistler heat-flux instability in solar wind conditions. Astrophys. J. Lett. 2019, 882, L8. [CrossRef]

24. Micera, A.; Zhukov, A.N.; López, R.A.; Innocenti, M.E.; Lazar, M.; Boella, E.; Lapenta, G. Particle-in-cell simulation of whistler heat-flux instabilities in the solar wind: Heat-flux regulation and electron halo formation. Astrophys. J. Lett. 2020, 903 , L23.

25. Jeong, S.Y.; Verscharen, D.; Wicks, R.T.; Fazakerley, A.N. A Quasi-linear Diffusion Model for Resonant Wave-Particle Instability in Homogeneous Plasma. Astrophys. J. 2020, 902, 128. [CrossRef]

26. Melrose, D.B. Instabilities in Space and Laboratory Plasmas; Cambridge University Press: Cambridge, UK, 1986. [CrossRef]

27. Shaaban, S.M.; Lazar, M.; Yoon, P.H.; Poedts, S.; López, R.A. Quasi-linear approach of the whistler heat-flux instability in the solar wind. Mon. Not. R. Astron. Soc. 2019, 486, 4498-4507. [CrossRef]

28. Alterman, B.L.; Kasper, J.C.; Stevens, M.L.; Koval, A. Comparison of alpha particle and proton beam differential flows in collisionally young solar wind. Astrophys. J. 2018, 864, 112. [CrossRef]

29. Vedenov, A.A.; Velikhov, E.P.; Sagdeev, R.Z. Nonlinear oscillations of rarified plasma. Nucl. Fusion 1961, 1, 82. (In Russian) [CrossRef]

30. Kennel, C.F.; Engelmann, F. Velocity space diffusion from weak plasma turbulence in a magnetic field. Phys. Fluids 1966, 9 , 2377. [CrossRef]

31. Harding, J.C.; Cairns, I.H.; Melrose, D.B. Electron-Langmuir wave resonance in three dimensions. Phys. Plasmas 2020, $27,020702$. [CrossRef]

32. Melrose, D.B.; Harding, J.C.; Cairns, I.H. Type-III electron beams: 3D quasilinear effects. Sol. Phys. 2021, 296, 42. [CrossRef]

33. Gary, S.P.; Yin, L.; Winske, D.; Ofman, L.; Goldstein, B.E.; Neugebauer, M. Consequences of proton and alpha anisotropics in the solar wind: Hybrid simulations. J. Geophys. Res. Space Phys. 2003, 108, 1-11. [CrossRef]

34. Ďurovcová, T.; Šafránková, J.; Němeček, Z. Evolution of relative drifts in the expanding solar wind: Helios observations. Sol. Phys. 2019, 294, 97. [CrossRef]

35. Liu, W.; Zhao, J.; Xie, H.; Yao, Y.; Wu, D.; Lee, L.C. Electromagnetic proton beam instabilities in the inner heliosphere: Energy transfer rate, radial distribution, and effective excitation. Astrophys. J. 2021, 920, 158. [CrossRef] 